\title{
A Supervised Model for Diabetes Divination
}

\author{
Sweta Jain ${ }^{1 *}$ \\ Assistant Professor, Department of Computer Science and Engineering, Shri Ramdeobaba \\ College of Engineering and Management, Nagpur, Maharashtra, India.
}

\section{ABSTRACT}

Diabetes is a most significant and sensitive metabolic disorder. It can disrupt entire metabolism of the body. The undiagnosed diabetes may exaggerate the risk of many other diseases too. It can cause problems such as weakness, stress and low interest in routine work. People with diabetes are among the most susceptible to novel corona virus (COVID19) infection and may grieve from serious lungs infections, difficulties in breathing, or even death. It is a motive of world-wide apprehension as the cases are intensifying hastily. In the current scenario with the advent and outstretched growth of Machine learning (ML), the computer assisted automatic disease diagnosis in healthcare segment is speedily growing. The present research aims to apply supervised ML practises on Pima Indian Diabetes (PID) corpus to diagnose disease for the females and assist the doctors and health care professionals. The empirical research is carried out using three different predictive models namely support vector machine (SVM) with linear, RBF, Polynomial, sigmoid functions, k-nearest neighbor (k-NN), and Random forest (RF).The random forest model has delivered an improved accuracy of $76 \%$.The performance of three models is measured by using accuracy, precision and recall.

KEY WORDS: DIABETES, MACHINE LEARNING, SUPPORT VECTOR MACHINE (SVM), K-NEAREST NEIGHBOR, RANDOM FOREST.

\section{INTRODUCTION}

Diabetes is a cluster of severe disorders demarcated by a high blood sugar level. The unhealthy lifestyle and lack of self-care are also additional causes. The common indicators of this disease comprise recurrent urination, increased thirst, weakness and augmented appetite. If the diabetes is left uncured, it may consequence into many other severe problems or even death. It is observed that this disease is growing with a massive pace. Several Millions of the masses are affecting their life.It is noticed that this disease has its strong roots in the low and average earning populations. The healthcare services are

\section{ARTICLE INFORMATION}

Received 11th Oct 2020 Accepted after revision 24th Dec 2020 Print ISSN: 0974-6455 Online ISSN: 2321-4007 CODEN: BBRCBA

Thomson Reuters ISI Web of Science Clarivate Analytics USA and Crossref Indexed Journal

\section{Clarivate
Analytics}

NAAS Journal Score 2020 (4.31)

A Society of Science and Nature Publication,

Bhopal India 2020. All rights reserved.

Online Contents Available at: http//www.bbrc.in/

Doi: $h$ ttp://dx.doi.org/10.21786/bbrc/13.14/73 also expensive and it may not be easily approachable in remote places like small towns and distance places. This disease may also boost the mortality rate of a country in conjunction with COVID-19.The country like India with massive young population needs strong support system for healthcare industry to provide affordable services needed for routine checkups and treatment of this deisease.As it has been noticed that this is common amongst higher and old age people, so we can provide substantial medical facility and advice in online mode for such masses in the country. Finally, it may be worth to mention that it may affect our economic expenses on healthcare budget.

Since the past two decades Machine learning and deep learning has enflamed extensive success in healthcare domain. It aimed to assist the medical professionals in computer assisted diagnostic of many diseases like breast cancer, skin cancer, lung cancer, diabetic retinopathy, brain disorders and many more. In medical diagnosis, data is sometimes limited, imbalance and incomplete. The machine learning algorithms can process multimodal, 
complex, high dimensional data, noisy and incomplete data to extract meaningful patterns from the data to analyze specific diagnosis application. The aim of the proposed research is to develop a predictive model for assistance in diabetes prediction using standard dataset.

Numerous researchers have performed exploratory data analysis for diabetes perdition. The existing studies shown that the corpus is still very limited and include only female patient's details. These studies provides new insights for amplifying the correctness and delicacy of all the existing methods. None of these methods have still shown a perfection in the operation and its real life insinuations, so as to apply it for enormous and effective
outcome.In the presented research the supervised model is instigated to perform exploratory data analysis and predict whether a person is diabetic (outcome $=1$ ) or not (Outcome $=0$ ). The dataset include eight predictor variables and one response variable. This corpus has a collection of all female records with age ranging between 21 to 81 years.This empirical research is made using three recurrently used models.The attributes in the corpus are representing input and output pair for each instance. This study is instigated on all the attributes. There is no evidence observed for confiscating any specific information during categorization.

Related Work: The related literature surveyed on diabetes prediction is summarized in Table1.0nly representative studies are highlighted with their key findings.

Table 1. Literature Studies based on ML models are summarized.

\begin{tabular}{|c|c|c|c|}
\hline Reference & Models & $\begin{array}{l}\text { Accuracy } \\
\text { Achieved (\%) }\end{array}$ & Finding \\
\hline $\begin{array}{l}\text { Harleen Kaur, } \\
\text { Vinita Kumari } \\
\text { (2018)[1] }\end{array}$ & $\begin{array}{l}\text { SVM,k-NN,ANN } \\
\text { Multifactor dimensionality } \\
\text { reduction (MDR) }\end{array}$ & $\begin{array}{l}\text { Ranges between } \\
83-89 \text { for various } \\
\text { classifier }\end{array}$ & $\begin{array}{l}\text { This study highlights that MDR } \\
\text { approach and } \mathrm{k}=13 \text { in } \mathrm{k}-\mathrm{NN} \text { has } \\
\text { Shown an enhanced effects. }\end{array}$ \\
\hline $\begin{array}{l}\text { Deepti Sisodia, } \\
\text { Dilip Singh Sisodia } \\
(2018)[2]\end{array}$ & $\begin{array}{l}\text { Decision Tree(DT), } \\
\text { and Naive Bayes }\end{array}$ & $\begin{array}{l}\text { Naive Bayes: } \\
76.30 \\
\text { SVM }=65.10 \\
\text { Decision Tree: } \\
73.82\end{array}$ & $\begin{array}{l}\text { The presented models have achieved } \\
\text { an average degree of } \\
\text { Correctness. }\end{array}$ \\
\hline $\begin{array}{l}\text { Deeksha } \\
\text { Harika Raju, } \\
\text { B.K Tripathy } \\
(2017)[3]\end{array}$ & $\begin{array}{l}\text { SVM, } \\
\text { Deep Neural Networks, } \\
\text { Hybrid Deep Leaming }\end{array}$ & $\begin{array}{l}\text { SVM: } 77.34 \\
\text { DNN: } 78.12 \\
\text { Hybrid Deep } \\
\text { Leaming: } 80.34\end{array}$ & $\begin{array}{l}\text { This study has applied deep } \\
\text { leaming model on a limited } \\
\text { Corpus. }\end{array}$ \\
\hline $\begin{array}{l}\text { Prema N S } \\
(2019)[4]\end{array}$ & $\begin{array}{l}\text { Logistic Regression,DT } \\
\text { SVM,RF,k-NN,Naïve } \\
\text { Bayes,Ada Boost, voting } \\
\text { classifier }\end{array}$ & 75.8 to 79.53 & $\begin{array}{l}\text { Highest accuracy is achieved for } \\
\text { Ensemble model. }\end{array}$ \\
\hline $\begin{array}{l}\text { Dilip Kumar } \\
\text { Choubey } \\
(2017)[5]\end{array}$ & Naïve Bayes(NB) & 78.69 & $\begin{array}{l}\text { Genetic Algorithm is used for } \\
\text { attribute selection and NB is } \\
\text { Used for classification. } \\
\text { Computation cost is minimized. }\end{array}$ \\
\hline $\begin{array}{l}\text { Uswa Ali Zia } \\
(2017)[6]\end{array}$ & Naive Bayes,DT,k-NN & $\begin{array}{l}74.84 \text { to } \\
94.44\end{array}$ & $\begin{array}{l}\text { DT algorithms J4S and Jgraft } \\
\text { Outperformed over other models. } \\
\text { The result are obtained after } \\
\text { Bootstrapping. }\end{array}$ \\
\hline $\begin{array}{l}\text { V. Anuja Kumari, } \\
\text { R.Chitra } \\
(2018)[7]\end{array}$ & SVM & 78 & $\begin{array}{l}\text { The focus is only limited to the } \\
\text { One of the model. }\end{array}$ \\
\hline
\end{tabular}

Figure 1: The Proposed System flow for Predictive Learning Model

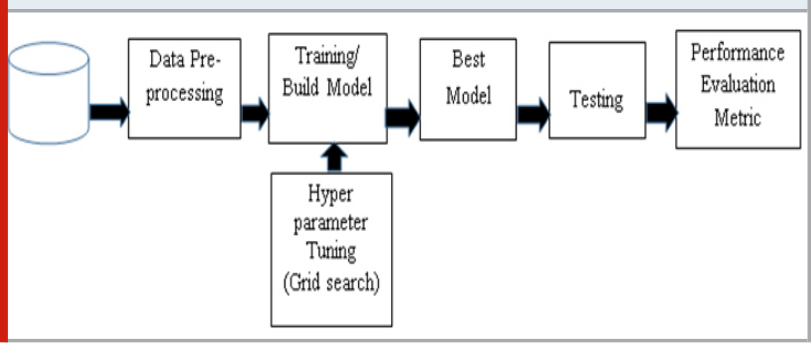

\section{MATERIAL AND METHODS}

Dataset: This study uses an open access corpus for computer assisted analysis. This dataset has total eight independent/Predictor variables and one dependent or response variable. The main impetus of using this dataset is to accomplish predictive analysis using popular classifiers. This dataset embraces 768 female patient's instances in the age ranging between 21 and 81 years. The corpus consist of 268 diabetic records and 500 non diabetic record. This dataset is imbalance. The train test split used in the presented work is 70-30\% respectively. As this dataset is imbalance, thus stratified sampling is used during train test split. System Representation: The suggested system steps are revealed in Fig.1.

Dealing with Data: It is a well-known fact that the data pre-processing is an important and relevant step for understanding the insights of the data. In real-life datasets such as medical diagnosis scenario there can be restricted data, and inequity data. To improve the predictive power of the model it is necessary to improve the data significance. It is obligatory to pre-process the data to achieve good performance of the system. 
Machine Learning Algorithms: In this presented work the main idea is to apply recent advancements thriving in the technology for making better service in the society and help the masses to prevent from severe medical problems. These classifiers have been realised using python sci-kit learn library.The SVM classifier is applied with linear, polynomial, RBF, and sigmoid kernel. An experimental approach is used to determine the $\mathrm{k}$ value for an improved testing accuracy.

Table 2. Comparisons of performance evaluation measures of various
models.
\begin{tabular}{|l|c|c|c|c|c|}
\hline & Accuracy & Precision & Recall & F1-Score & AUC \\
\hline k-NN & 0.74 & 0.67 & 0.52 & 0.58 & 0.74 \\
\hline SVM (Linear Kernel) & 0.73 & 0.65 & 0.49 & 0.56 & 0.83 \\
\hline SVM(Polynomial Degree=3) & 0.74 & 0.74 & 0.40 & 0.52 & 0.81 \\
\hline SVM(RBF kernel) & 0.74 & 0.72 & 0.44 & 0.55 & 0.82 \\
\hline SVM (Sigmoid Kernel) & 0.51 & 0.14 & 0.07 & 0.10 & 0.68 \\
\hline Random Forest(RF) & 0.76 & 0.70 & 0.54 & 0.61 & 0.83 \\
\hline
\end{tabular}

Figure 2: k-NN (k=7)

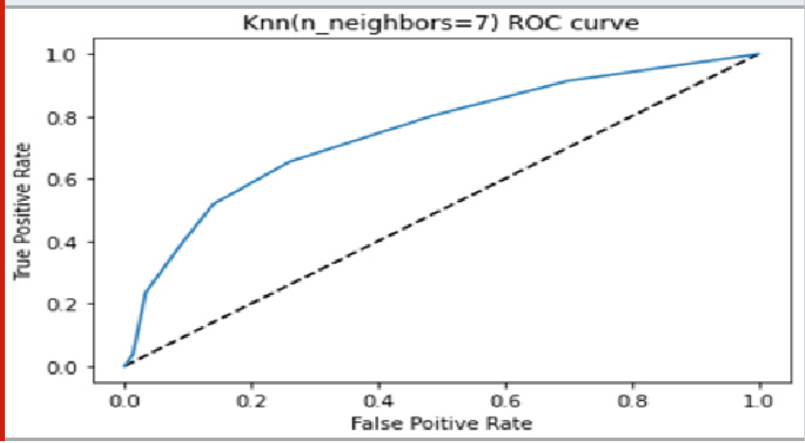

Figure 4: SVM (Linear) and RF

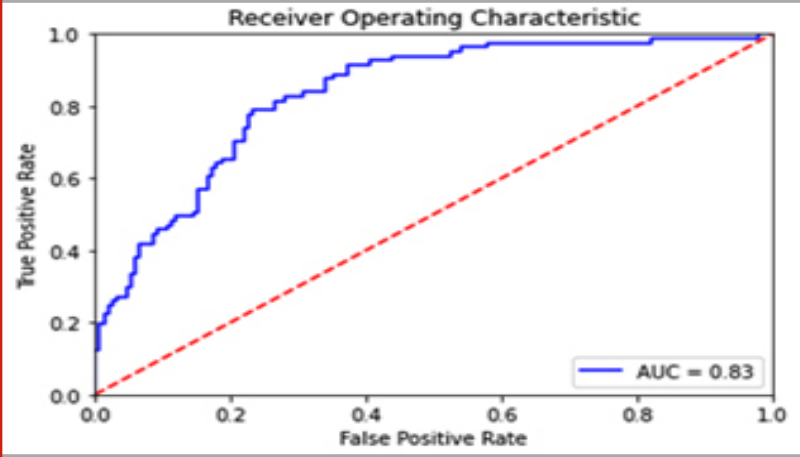

K-NN: In this study, the instance based method is used for the categorization of affected and non-affected females. The most crucial task in its implementation is to determine the value of $\mathrm{k}$.In this proposed work a grid search method is applied to find the parameter. This algorithm predicts the classification result, for a new test instance based on opinion or voting of neighbors in the nearest proximity. The algorithm uses distance measures such as Euclidean, Hamming, and Manhattan for locating the nearest neighbor. This is lethargic approach, as the Instances are simply stored in the memory in the initial step.
Figure 3: Testing accuracy is good for $k-\mathrm{NN}(\mathrm{k}=7)$

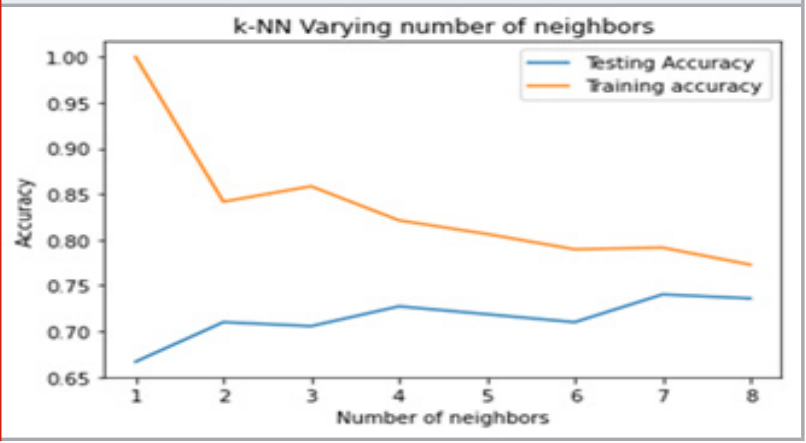

Figure 5: SVM (Polynomial)

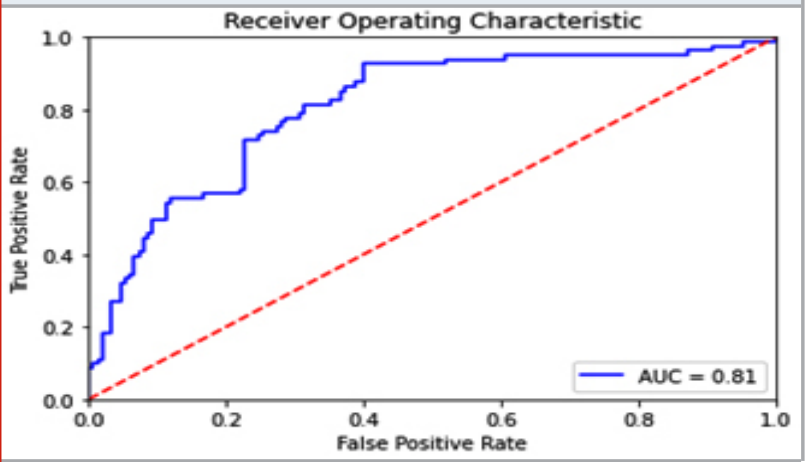

SVM: This model is remarkably used for the classification in the medical applications. The linear SVM uses maximum margin hyper plane for the separation of the data. The SVM can model linear as well as nonlinear data. The proposed work is implemented with Linear, polynomial (degree=3), RBF, and sigmoid kernel functions.SVM works effectively in high dimensional space. This model is still operative even if the dimension in the data is bigger than the total number of instances in the corpus. 
Figure 6: SVM (RBF)

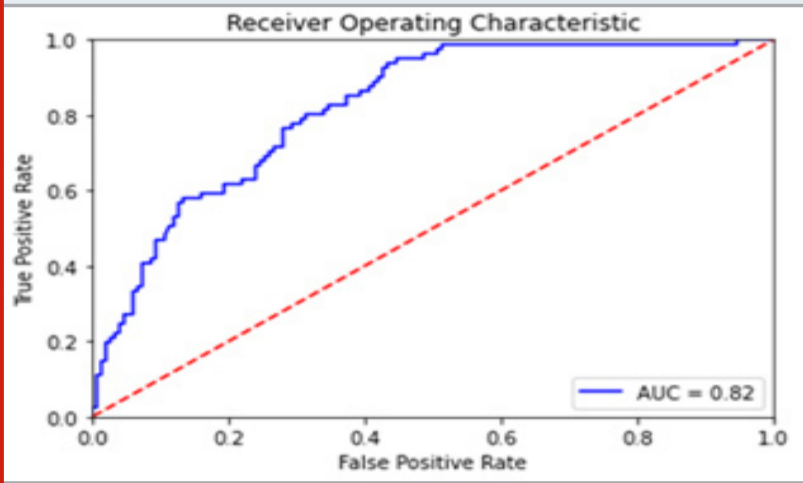

Figure 7: SVM (sigmoid)

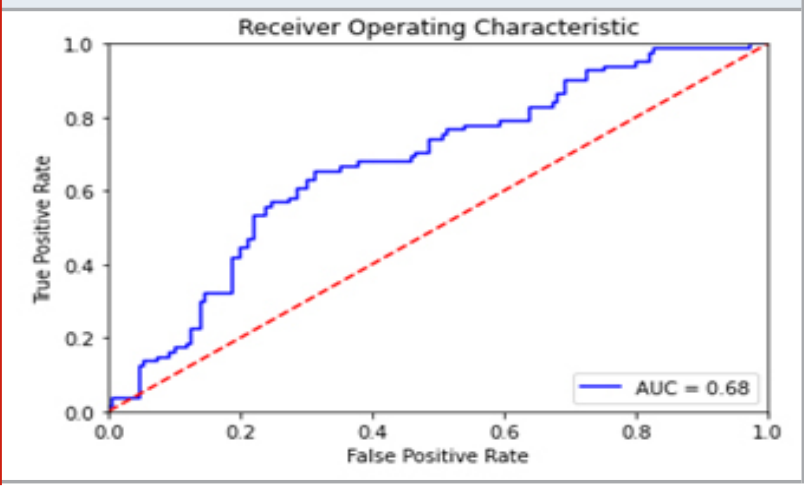

Random Forest: This learning algorithm falls into the category of ensemble learning method. This model builds its inference by combining the impact of several decision trees. This model is used in two class categorization as well as multiple class categorization. The accuracy level of this ensemble model is generally higher.

\section{Performance Evaluation metric}

The experimented models are assessed on the wellknown evaluation metric. The details are also outlined in the Table2.

\section{RESULTS AND DISCUSSION}

The empirical outputs of the proposed system are summarized in Table2.The Random Forest classifier outperformed in diabetes diagnosis. The ROC curves are highlighted for SVM, k-NN, and RF. The SVM Model with RBF and polynomial kernels have shown an improved accuracy over sigmoid kernel.

In medical diagnosis applications evaluation measures such as Precision and Recall are very important. Fig2 to Fig.7 represents the receiver operating characteristics (ROC) curve for k-NN, SVM with different kernels, and Random Forest.The Precision of SVM model with polynomial and RBF kernel is better than other models.
The k-NN algorithm produced an improved testing accuracy for $\mathrm{k}=7$ neighbors as shown in fig.3.The SVM with Polynomial degree $=3$ highlights largest precision value as compared to other models. Even if the degree of polynomial is increased to a lager value than it has been noticed that the generalization power of the model is not noteworthy.

\section{CONCLUSION}

The presented study conversed the accomplishment of three ML models to perform diabetes diagnosis using all the features in the structured dataset. It focuses on assisting the early diabetes diagnosis for females. The analysis of presented experimental research outlines that the random forest model performed better than SVM and k-NN with highest accuracy of 76\%.The SVM model also achieved a nearly good accuracy with linear, polynomial and RBF kernel.The SVM with gaussian exhibited the worst accuracy. This study is limited to the diabetes prediction using structured dataset and it contains all instances of females. The new corpus may be collected for processing male patient's information. In future more prominence may be given to process high dimensional unstructured data using deep learning models.

\section{REFERENCES}

D. Sisodia and D. S. Sisodia, "Prediction of Diabetes using Classification Algorithms," Procedia Comput. Sci., vol. 132, no. Iccids, pp. 1578-1585, 2018, doi: 10.1016/j. procs.2018.05.122.

D. Kaul, H. Raju, and B. K. Tripathy, "Comparative Analysis of Pure and Hybrid Machine Learning Algorithms for Risk Prediction of Diabetes Mellitus," Helix, vol. 7, no. 5, pp. 2029-2033, 2017, doi: 10.29042/2017-2029-2033.

D. K. Choubey, S. Paul, S. Kumar, and S. Kumar, "Classification of pima indian diabetes dataset using naive bayes with genetic algorithm as an attribute selection," Commun. Comput. Syst. - Proc. Int. Conf. Commun. Comput. Syst. ICCCS 2016, no. November, pp. 451-455, 2017, doi: 10.1201/9781315364094-82.

K. and Chitra, "Classification Of Diabetes Disease Using Support Vector Machine,” vol. 3, no. 2, pp. 1797-1801, 2018, [Online]. Available: https://www.researchgate.net/ publication/320395340.

H. Kaur and V. Kumari, "Predictive modelling and analytics for diabetes using a machine learning approach," Appl. Comput. Informatics, no. xxxx, pp. 0-5, 2019, doi: 10.1016/j.aci.2018.12.004.

N. S. Prema, V. Varshith, and J. Yogeswar, "Prediction of diabetes using ensemble techniques," Int. J. Recent Technol. Eng., vol. 7, no. 6, pp. 203-205, 2019.

U. Ali Zia and N. Khan, "Predicting Diabetes in Medical Datasets Using Machine Learning Techniques," Int. J. Sci. Eng. Res., vol. 8, no. 5, pp. 1538-1551, 2017, [Online]. Available: http://www.ijser.org. 